\title{
THE ASYMPTOTIC BEHAVIOR OF UNIVALENT FUNCTIONS
}

\author{
HU KE AND DONG XINHAN
}

\begin{abstract}
We generalize Bazilevic's Theorem and provide an alternative proof of an important theorem of Hamilton.
\end{abstract}

1. Introduction. Let $f(z)=z+\sum_{n=2}^{\infty} a_{n} z^{n} \in S$. B. G. Eke [2] defined a class of functions $S\left(\theta_{1}, \theta_{2}, \ldots, \theta_{k}\right)$ as follows: $f \in S$ and there exist $C>0, \delta>0$, and a sequence $r_{n} \rightarrow 1$ such that $[7]$

$$
\left(1-r_{n}\right)^{2 / k}\left|f\left(r_{n} e^{i \theta_{n}^{(\nu)}}\right)\right| \geq C, \quad \nu=1,2, \ldots, k, n=1,2, \ldots,
$$

for

$$
\delta<\theta_{n}^{(\nu)}-\theta_{n}^{(\mu)}<2 \pi-\delta, \quad 1 \leq \nu<\mu \leq k .
$$

The following is from works of Hayman [4] and Eke [2].

THEOREM A. If $f(z)=z+\sum_{n=2}^{\infty} a_{n} z^{n} \in S$, then

$$
\lim _{n \rightarrow \infty}|| a_{n}|-| a_{n+1} \| \leq 1
$$

except $f \in S\left(\theta_{1}, \theta_{2}\right)$.

In 1982 Hamilton [3] obtained a beautiful result:

THEOREM B. If $f(z)=z+\sum_{n=2}^{\infty} a_{n} z^{n} \in S\left(\theta_{1}, \theta_{2}\right)$, then

$$
\varlimsup_{n \rightarrow \infty}|| a_{n}|-| a_{n+1} \| \leq 1 \text {. }
$$

Here we generalize Bazilevic's Theorem and provide an alternate proof of this important theorem of Hamilton.

2. A generalization of Bazilevic's Theorem. We now define

$$
\log \frac{f(z)}{z}=2 \sum_{n=1}^{\infty} \gamma_{n} z^{n}
$$

the Grunsky expansion

$$
G(z, \zeta)=\log \frac{1 / f(z)-1 / f(\varsigma)}{1 / z-1 / \zeta}=-\sum_{n=1}^{\infty} A_{n}\left(\frac{1}{\zeta}\right) z^{n},
$$

and the Faber polynomials for $F(\xi)=1 / f(z)$ with $\xi=1 / z$

$$
\Phi_{n}\left(\frac{1}{f(z)}\right)=\frac{z^{-n}}{n}+A_{n}\left(\frac{1}{z}\right) \quad \text { and } \quad 2 \gamma_{n}=\Phi_{n}(0) .
$$

Received by the editors March 10, 1985 and, in revised form, February 24, 1986.

1980 Mathematics Subject Classification (1985 Revision). Primary 30C50; Secondary 30C55. 
The Grunsky inequality states that

$$
\sum_{n=1}^{\infty} n\left|\sum_{\nu=1}^{k} A_{n}\left(\frac{1}{z_{\nu}}\right)\right|^{2} \leq \sum_{\mu, \nu=1}^{k} \log \frac{1}{\left|1-z_{\mu} \bar{z}_{\nu}\right|}
$$

We have

THEOREM 1. If $f(z) \in S\left(\theta_{1}, \theta_{2}, \ldots, \theta_{k}\right)$, then

$$
\sum_{n=1}^{\infty} n\left|\gamma_{n}-\frac{1}{k} \sum_{\nu=1}^{k} e^{-i n \theta_{\nu}}\right|^{2} \leq \frac{1}{2} \log \frac{1}{C}+\frac{1}{2 k} \sum_{\mu \neq \nu} \log \frac{2}{\left|e^{i \theta_{\mu}}-e^{i \theta_{\nu}}\right|^{2}}
$$

where $C$ is determined by (1.1) and $\theta_{\nu}$ is one of the limit points of $\left\{\theta_{n}^{(\nu)}\right\}$ for each $\nu(\nu=1,2, \ldots, k)$.

The case $k=1$ is due to Bazilevic [1].

ProOF. Notice the following identity:

$$
\begin{aligned}
&\left|\sum_{\nu=1}^{k}\left(A_{n}\left(\frac{1}{z_{\nu}}\right)-\frac{\bar{w}_{\nu}^{n}}{n}\right)\right|^{2} \\
&=\left|\sum_{\nu=1}^{k} A_{n}\left(\frac{1}{z_{\nu}}\right)\right|^{2}-\frac{2}{n} \operatorname{Re} \sum_{\mu, \nu=1}^{k} A_{n}\left(\frac{1}{z_{\nu}}\right) w_{\mu}^{n}+\left|\sum_{\nu=1}^{k} \frac{w_{\nu}^{n}}{n}\right|^{2},
\end{aligned}
$$

and use (2.2) and the classical inequality

$$
\left|\frac{z f^{\prime}(z)}{f(z)}\right| \leq \frac{1+|z|}{1-|z|} \quad \text { for }\left|z_{\nu}\right|=r_{n}
$$

to deduce

$$
\begin{aligned}
\sum_{n=1}^{\infty} n\left|\sum_{\nu=1}^{k} A_{n}\left(\frac{1}{z_{\nu}}\right)-\frac{\bar{z}_{\nu}}{n}\right|^{2} \leq & 2 \sum_{\mu, \nu=1}^{k}\left\{\log \frac{1}{\left|1-z_{\mu} \bar{z}_{\nu}\right|}+\operatorname{Re} G\left(z_{\mu}, z_{\nu}\right)\right\} \\
\leq & 2 \sum_{\nu=1}^{k} \log \frac{\left|f^{\prime}\left(z_{\nu}\right)\right|\left|z_{\nu}\right|^{2}}{\left|f\left(z_{\nu}\right)\right|^{2}\left(1-\left|z_{\nu}\right|^{2}\right)} \\
& +2 \sum_{\mu \neq \nu} \log \frac{\left|1 / f\left(z_{\mu}\right)-1 / f\left(z_{\nu}\right)\right|\left|z_{\mu} z_{\nu}\right|}{\left|z-z_{\nu}\right|\left|1-z_{\mu} \bar{z}_{\nu}\right|} \\
\leq & 2 \sum_{\nu=1}^{k} \log \frac{1}{\left(1-r_{m}\right)^{2}\left|f\left(z_{\nu}\right)\right|} \\
& +2 \sum_{\mu \neq \nu} \log \frac{1 /\left|f\left(z_{\mu}\right)\right|+1 /\left|f\left(z_{\nu}\right)\right|}{\left|z_{\mu}-z_{\nu}\right|\left|1-z_{\mu} \bar{z}_{\nu}\right|} \\
\leq & 2 \sum_{\nu=1}^{k} \log \frac{\left(1-r_{m}\right)^{2 / k}}{C\left(1-r_{m}\right)^{2}} \\
& +2 \sum_{\mu \neq \nu} \log \frac{2\left(1-r_{m}\right)^{2 / k}}{C\left|z_{\mu}-z_{\nu}\right|^{2}}
\end{aligned}
$$


Now as $z_{m}^{(\nu)} \rightarrow e^{i \theta_{\nu}}, A_{n}$ tends to $\Phi_{n}(0)-e^{-i n \theta_{\nu}} / n=2 \gamma_{n}-e^{-i n \theta_{\nu}} / n$. Thus

$$
\sum_{n=1}^{\infty} n\left|2 k \gamma_{n}-2 \sum_{\nu=1}^{k} \frac{e^{-i n \theta_{\nu}}}{n}\right|^{2} \leq 2 k^{2} \log \frac{1}{C}+2 \sum_{\mu \neq \nu} \log \frac{2}{\left|e^{i \theta_{\mu}}-e^{i \theta_{\nu}}\right|^{2}}
$$

Dividing by $4 k^{2}$ on both sides we complete the proof of Theorem 1 .

THEOREM 2. For all $0<p<\infty$, if $f(z) \in S\left(\theta_{1}, \theta_{2}, \ldots, \theta_{k}\right)$, then

$$
\frac{f(z)}{z} \prod_{\nu=1}^{n}\left(1-z e^{-i \theta_{\nu}}\right)^{2 / k} \in H^{p}
$$

and there exists $F_{p}(t) \in L[0,2 \pi]$ such that

$$
\begin{array}{r}
f(z)=z \prod_{\nu=1}^{k}\left(1-z e^{-i \theta_{\nu}}\right)^{-2 / k}\left\{\frac{1}{2 \pi} \int_{0}^{2 \pi} \frac{1-r^{2}}{1-2 r \cos (\theta-t)+r^{2}} F_{p}(t) d t\right\}^{1 / p} \\
z=r e^{i \theta} .
\end{array}
$$

The case $k=1$ is proved in [5].

Proof. For $p \in(0, \infty)$, let

$$
\begin{gathered}
\varphi(z)=\left\{\frac{f(z)}{z} \prod_{\nu=1}^{k}\left(1-z e^{-i \theta_{\nu}}\right)^{2 / k}\right\}^{p / 2}=\sum_{n=0}^{\infty} D_{n}(p) z^{n}, \quad D_{0}=1 \\
\log \varphi(z)=\frac{p}{2} \sum_{n=1}^{\infty}\left(2 \gamma_{n}-\frac{2}{k} \sum_{\nu=1}^{k} \frac{e^{-i n \theta_{\nu}}}{n}\right) z^{n}
\end{gathered}
$$

From the Milin [5] inequality and Theorem 1 we conclude

$$
\begin{aligned}
\frac{1}{2 \pi} \int_{0}^{2 \pi}|\varphi(z)|^{2} d \theta & =\sum_{n=0}^{\infty}\left|D_{n}(p)\right|^{2} r^{2 n} \\
& \leq \exp \left\{\frac{p^{2}}{4} \sum_{n=1}^{\infty} n\left|2 \gamma_{n}-\frac{2}{k} \sum_{\nu=1}^{k} \frac{e^{-i n \theta_{\nu}}}{n}\right|^{2} r^{2 n}\right\} \\
& \leq \exp \left\{\frac{p^{2}}{4}\left[2 \log \frac{1}{C}+\frac{2}{k^{2}} \sum_{\mu \neq \nu} \log \frac{2}{\left|e^{i \theta_{\mu}}-e^{i \theta_{\nu}}\right|^{2}}\right]<\infty\right.
\end{aligned}
$$

Consequently,

$$
\varphi^{2}=\left\{\frac{f(z)}{z} \prod_{\nu=1}^{k}\left(1-z e^{-i \theta_{\nu}}\right)^{2 / k}\right\}^{p} \in H^{1}
$$

and $f(z)$ has the integral representation as in (2.7). 
3. An application of Theorem 1. We now prove Theorem B. Let $f(z)=$ $z+\sum_{n=2}^{\infty} a_{n} z^{n} \in S\left(\theta_{1}, \theta_{2}\right)$. Then

$$
\begin{gathered}
\left(1-z e^{-i \theta_{1}}\right) \frac{f(z)}{z}=1+\sum_{n=1}^{\infty}\left(a_{n+1}-e^{-i \theta_{1}} a_{n}\right) z^{n} \\
\log \left[\left(1-z e^{-i \theta_{1}}\right) \frac{f(z)}{z}\right]=\sum_{n=1}^{\infty}\left(2 \gamma_{n}-\frac{e^{-i n \theta_{1}}}{n}\right) z^{n} \\
\log \left[\left(1-z e^{-i \theta_{1}}\right)\left(1-z e^{-i \theta_{2}}\right) \frac{f(z)}{z}\right]=\sum_{n=1}^{\infty}\left(2 \gamma_{n}-\frac{e^{-i n \theta_{1}}}{n}-\frac{e^{-i n \theta_{2}}}{n}\right) z^{n} .
\end{gathered}
$$

Replacing $z$ by $\sqrt{r} z$ and using the Milin inequality [6] we obtain

$$
\begin{aligned}
& T_{n}^{2}=\left\|a_{n}|-| a_{n+1}\right\|^{2} \leq\left|a_{n+1}-a_{n} e^{-i \theta_{1}}\right|^{2} \\
& \leq r^{-n} \exp \left\{\sum_{k=1}^{n} k\left|2 \gamma_{k}-\frac{e^{-i k \theta_{1}}}{k}\right|^{2} r^{k}-\sum_{k=1}^{n} \frac{1}{k}\right\} \\
& \leq r^{-n} \exp \left\{\sum_{k=1}^{\infty} k\left|2 \gamma_{k}-\frac{e^{-i k \theta_{1}}}{k}-\frac{e^{-i k \theta_{2}}}{k}\right|^{2} r^{k}+\sum_{k=1}^{n} \frac{1}{k} r^{k}-\sum_{k=1}^{n} \frac{1}{k}\right. \\
& \left.+2 \operatorname{Re} \sum_{k=1}^{n} r^{k} e^{i k \theta_{2}}\left[2 \gamma_{k}-\frac{e^{-i k \theta_{1}}}{k}-\frac{e^{-i k \theta_{2}}}{k}\right]\right\} \\
& \leq r^{-n} \exp \left\{\sum_{k=1}^{\infty} k\left|2 \gamma_{k}-\frac{e^{-i k \theta_{1}}}{k}-\frac{e^{-i k \theta_{2}}}{k}\right|^{2}\right. \\
& +2 \operatorname{Re} \sum_{k=1}^{\infty} r^{k} e^{i k \theta_{2}}\left[2 \gamma_{k}-\frac{e^{-i k \theta_{1}}}{k}-\frac{e^{-i k \theta_{2}}}{k}\right] \\
& \left.-2 \operatorname{Re} \sum_{k=n+1}^{\infty} r^{k} e^{i k \theta_{2}}\left|2 \gamma_{k}-\frac{e^{-i k \theta_{1}}}{k}-\frac{e^{-i k \theta_{2}}}{k}\right|\right\} \\
& \leq r^{-n} \exp \left\{\sum_{k=1}^{\infty} k\left|2 \gamma_{k}-\frac{e^{-i k \theta_{1}}}{k}-\frac{e^{-i k \theta_{2}}}{k}\right|^{2}\right. \\
& +2 \log \frac{(1-r)\left|1-r e^{i\left(\theta_{1}-\theta_{2}\right)} \| f\left(r e^{i \theta_{2}}\right)\right|}{r} \\
& \left.+2 \sum_{k=n+1}^{\infty} r^{k}\left|2 \gamma_{k}-\frac{e^{-i k \theta_{1}}}{k}-\frac{e^{-i k \theta_{2}}}{k}\right|\right\} \text {. }
\end{aligned}
$$

By Theorem 1,

$$
\sum_{k=1}^{\infty} k\left|2 \gamma_{k}-\frac{e^{-i k \theta_{1}}}{k}-\frac{e^{-i k \theta_{2}}}{k}\right|^{2}<\infty
$$


Therefore,

$$
\begin{aligned}
& \sum_{k=n+1}^{\infty} r^{k}\left|2 \gamma_{k}-\frac{e^{-i k \theta_{1}}}{k}-\frac{e^{-i k \theta_{2}}}{k}\right| \\
& \leq\left\{\sum_{k=n+1}^{\infty} k\left|2 \gamma_{k}-\frac{e^{-i k \theta_{1}}}{k}-\frac{e^{-i k \theta_{2}}}{k}\right|^{2} \sum_{k=n+1}^{\infty} \frac{r^{2 k}}{k}\right\}^{1 / 2} \leq\left\{\frac{\varepsilon_{n}}{n(1-r)}\right\}^{1 / 2}
\end{aligned}
$$

where

$$
\varepsilon_{n}=\sum_{k=n+1}^{\infty} k\left|2 \gamma_{k}-\frac{e^{-i k \theta_{1}}}{k}-\frac{e^{-i k \theta_{2}}}{k}\right|^{2}
$$

Thus we have

$$
\begin{aligned}
T_{n}^{2} \leq & r^{-n} \exp \left\{\sum_{k=1}^{\infty} k\left|2 \gamma_{k}-\frac{e^{-i k \theta_{1}}}{k}-\frac{e^{-i k \theta_{2}}}{k}\right|^{2}\right. \\
& \left.+2 \log \frac{(1-r)\left|1-r e^{i\left(\theta_{2}-\theta_{1}\right)} \| f\left(r e^{i \theta_{2}}\right)\right|}{r}+2\left[\frac{\varepsilon_{n}}{n(1-r)}\right]^{1 / 2}\right\} \\
\leq & r^{-n} \exp \left\{S_{1}(r)+\omega_{1}(r)+2\left[\frac{\varepsilon_{n}}{n(1-r)}\right]^{1 / 2}\right\},
\end{aligned}
$$

where

$$
\begin{gathered}
S_{1}(r)=\sum_{k=1}^{\infty} k\left|2 \gamma_{k}-\frac{e^{-i k \theta_{1}}}{k}-\frac{e^{-i k \theta_{2}}}{k}\right|^{2}-\sum_{k=1}^{\infty} k\left|A_{k}\left(\frac{1}{r} e^{-i \theta_{1}}\right)-\frac{\left(r e^{-i \theta_{2}}\right)^{k}}{k}\right|^{2}, \\
\omega_{1}(r)=\sum_{k=1}^{\infty} k\left|A_{k}\left(\frac{1}{r} e^{-i \theta_{1}}\right)-\frac{\left(r e^{-i \theta_{2}}\right)^{k}}{k}\right|^{2}+2 \log \frac{(1-r)\left|1-r e^{i\left(\theta_{2}-\theta_{1}\right)} \| f\left(r e^{i \theta_{2}}\right)\right|}{r} .
\end{gathered}
$$

Since $\left|f\left(r e^{i \theta_{\nu}}\right)\right| \rightarrow \infty$ as $r \rightarrow 1(\nu=1,2)$, we conclude from (2.5) and Theorem 1 that $S_{1}(r) \rightarrow 0$ as $r \rightarrow 1$. Applying (2.3) with $k=1 \cdot$ we have

$$
\sum_{n=1}^{\infty} n\left|A_{n}\left(\frac{1}{r} e^{-i \theta_{1}}\right)-\frac{\left(r e^{-i \theta_{2}}\right)^{n}}{n}\right|^{2} \leq 2 \log \frac{(r /(1-r))\left[1 /\left|f\left(r e^{i \theta_{1}}\right)\right|+1 /\left|f\left(r e^{i \theta_{2}}\right)\right|\right]}{(1+r)\left|e^{i \theta_{1}}-e^{i \theta_{2}}\right|}
$$

Hence,

$$
\begin{gathered}
\omega_{1}(r) \leq 2 \log \frac{\left|1-r e^{i\left(\theta_{2}-\theta_{1}\right)}\right|\left(1+\left|f\left(r e^{i \theta_{2}}\right) / f\left(r e^{i \theta_{1}}\right)\right|\right)}{(1+r)\left|e^{i \theta_{1}}-e^{i \theta_{2}}\right|}=2 \delta_{1}(r), \\
T_{n}^{2} \leq r^{-n} \exp \left\{S_{1}(r)+2 \delta_{1}(r)+2\left[\frac{\varepsilon_{n}}{n(1-r)}\right]^{1 / 2}\right\} .
\end{gathered}
$$

Similarly, we have

$$
T_{n}^{2} \leq r^{-n} \exp \left\{S_{2}(r)+2 \delta_{2}(r)+2\left[\frac{\varepsilon_{n}}{n(1-r)}\right]^{1 / 2}\right\}
$$


where

$$
\begin{aligned}
S_{2}(r) & =\sum_{k=1}^{\infty} k\left|2 \gamma_{k}-\frac{e^{-i \theta_{1}}}{k}-\frac{e^{-i \theta_{2}}}{k}\right|^{2}-\sum_{k=1}^{\infty} k\left|A_{k}\left(\frac{1}{r} e^{-i \theta_{2}}\right)-\frac{\left(r e^{-i \theta_{1}}\right)^{k}}{k}\right|^{2}, \\
\delta_{2}(r) & =\log \frac{\left|1-r e^{i\left(\theta_{1}-\theta_{2}\right)}\right|\left(1+\left|f\left(r e^{i \theta_{1}}\right) / f\left(r e^{i \theta_{2}}\right)\right|\right)}{(1+r)\left|e^{i \theta_{1}}-e^{i \theta_{2}}\right|} .
\end{aligned}
$$

Thus

$$
T_{n}^{2} \leq r_{n}^{-n} \exp \left\{\eta(r)+2\left[\frac{\varepsilon_{n}}{n\left(1-r_{n}\right)}\right]^{1 / 2}\right\},
$$

where $\eta(r)=\min \left(S_{1}\left(r_{n}\right)+2 \delta_{1}\left(r_{n}\right), S_{2}\left(r_{n}\right)+2 \delta_{2}\left(r_{n}\right)\right)$, i.e., if $\left|f\left(r e^{i \theta_{1}}\right)\right| \geq\left|f\left(r e^{i \theta_{2}}\right)\right|$, then $\eta(r)=S_{1}(r)+2 \delta_{1}(r)$, otherwise $\eta(r)=S_{2}(r)+2 \delta_{2}(r)$. Therefore $\eta(r) \rightarrow 0$ as $r \rightarrow 1$. Take $r=1-\sqrt{\varepsilon_{n}} / n$ to deduce

$$
T_{n}^{2} \leq\left(1-\frac{\sqrt{\varepsilon_{n}}}{n}\right)^{-n} \exp \left\{\eta\left(r_{n}\right)+2 \varepsilon_{n}^{1 / 4}\right\},
$$

By Theorem 1, $\varepsilon_{n} \rightarrow 0$ as $n \rightarrow \infty$. It follows that $\varlimsup_{n \rightarrow \infty} T \leq 1$. The proof is complete.

4. Remark. In view of Theorem 2 it is natural to ask if

$$
\frac{f(z)}{z} \prod_{k=1}^{n}\left(1-z e^{-i \theta_{\nu}}\right)^{2 / k} \in \mathrm{BMO} A
$$

as $H_{p} \supset$ BMO $A \supset H_{\infty}$. However, we can find $f \in S(0, \pi)$, for example, not in BMO $A$.

$C-f(D)$ consists of 4 symmetric curves asymptotic to the $O Y$ axis at $\infty$. Considering 1/W plan:

Choose a curve such that $f$ is conformal at \pm 1 , so will have angular limit. Then there exists

$$
\lim _{r \rightarrow 1}(1-r)|f( \pm r)|=\alpha>0 .
$$

The cusp can be chosen so that $w_{n} \rightarrow \infty$ in the cusp $\operatorname{dist}\left(w_{n}, C-f(D)\right)=\infty$. The same will be true for $\left(1-z^{2}\right) f(z)=f_{0}$. Thus $f_{0}$ is not Bloch at $\pm i$, and therefore cannot be BMO $A$ (since $f_{0}$ is univalent near $\pm i$ ).

This example was provided by the referee.

5. Another remark. The contents of this paper have been presented to the meeting on Function Theory held in Nanjing in May 1985.

\section{REFERENCES}

1. I. E. Bazilevic, Coefficient dispersion of univalent functions, Mat. Sb. 68(110) (1965), 549-560. (Russian)

2. B. G. Eke, The asymptotic behaviour of a really mean valent functions, J. Analyse Math. 20(1967), 147-212.

3. D. H. Hamilton, The successive coefficients of univalent functions, J. London Math. Soc. 25(1982), 122-138.

4. W. K. Hayman, On successive coefficients of univalent functions, J. London Math. Soc. 38(1963), 228-243. 
5. Hu Ke, Some properties of univalent functions, J. Jiangxi Normal Univ. 3 (1986), 1-6.

6. I. M. Milin, Univalent functions and orthonormal systems, Izdat "Nauka", Moskow, 1971, pp. 3850 (Russian).

7. Ch. Pommerenke, Univalent functions, Vandenhoeck and Ruprecht, Göttingen, 1973, pp. 141145.

Department of Mathematics, JiAngXi Normal University, NANChang, China

Department of Mathematics, Hunan Normal University, Changsha, China 